\title{
The configuration and phase space of classical helium atom in 2D
}

\author{
A. Santhanam, M. Balakrishnan* and R. Arunchunai Annadurai \\ PG \& Research Dept. of Physics, Govt. Arts College, Tiruvannamalai - 606 603, India \\ mbkarunmathi_ro@yahoo.co.in*
}

\begin{abstract}
The classical three body problem of helium atom is studied numerically in two dimensions. The orbits and phase space of the electrons for different initial conditions are investigated by solving the equations of motion numerically. The phase space shows interesting result of band structure similar to the quantum mechanical result of the solids. The variation of auto-ionization with perturbation parameter is also established.
\end{abstract}

Keywords: Helium atom, torus, configuration space, phase space, auto-ionization, three body problem.

Introduction

Helium Atom is one of the most important three-body system under the influence of Coulomb force field and it is one of the most challenging non-integrable system. Some of the pioneers of quantum mechanics tried to solve this problem semi-classically (Bohr, 1913; Langumuir, 1921; Van Vleck, 1922). Also classical model of Helium atom was tried in the previous study (Yamamota \& kaneko, 1993). An attempt is made in the present work to study the phase space of the orbiting electrons in a helium atom. Two dimensional case of classical non-relativistic helium atom (with a nucleus and two orbiting electrons) problem is solved numerically.

\section{Materials and methods}

Dimensionless form of the equations of motion

The equation of motion for electron 1 is

$\mathrm{m}_{\mathrm{e}} \ddot{\mathrm{r}}_{1}=\frac{\mathrm{e}^{2}}{4 \pi \varepsilon_{\mathrm{o}}}\left(\frac{-2 \hat{\mathrm{r}}_{1}}{\mathrm{r}_{1}^{2}}+\frac{\hat{\mathrm{r}}_{12}}{\mathrm{r}_{12}^{2}}\right)$

Putting $\vec{R}_{n}=\frac{\vec{r}_{n}}{r_{o}}$, a dimensionless quantity and

replacing $\quad \hat{R}_{n}=\hat{r}_{n}$ and $r_{n}=r_{o} R_{n}$

we get, $\quad \frac{4 \pi \mathrm{r}_{\mathrm{o}}^{3} \varepsilon_{\mathrm{o}}}{\mathrm{e}^{2}} \mathrm{~m}_{\mathrm{e}} \frac{\mathrm{d}^{2} \overrightarrow{\mathrm{R}}_{1}}{\mathrm{dt}^{2}}=-\frac{2 \hat{\mathrm{R}}_{1}}{\mathrm{R}_{1}^{2}}+\frac{\hat{\mathrm{R}}_{12}}{\mathrm{R}_{12}^{2}}$

$\tau=\left(\frac{\mathrm{e}^{2}}{4 \pi \varepsilon_{\mathrm{o}} \mathrm{r}_{\mathrm{o}}^{3} \mathrm{~m}_{\mathrm{e}}}\right)^{1 / 2} \mathrm{t}$

where, $\mathrm{T}$ is a dimensionless time.

$\frac{\mathrm{d}^{2} \overrightarrow{\mathrm{R}}_{1}}{\mathrm{~d} \tau^{2}}=-\frac{2 \hat{\mathrm{R}}_{1}}{\mathrm{R}_{1}^{2}}+\frac{\hat{\mathrm{R}}_{12}}{\mathrm{R}_{12}^{2}}$

$\frac{\mathrm{d}^{2} \overline{\mathrm{R}}_{1}}{\mathrm{~d} \tau^{2}}=-\frac{2 \overline{\mathrm{R}}_{1}}{\mathrm{R}_{1}^{3}}+\frac{\overline{\mathrm{R}}_{12}}{\mathrm{R}_{12}^{3}}$

Similarly for the electron2,

Research article

CIndian Society for Education and Environment (iSee)

\begin{tabular}{|c|c|c|c|c|}
\hline $\begin{array}{c}\text { Initial } \\
\text { Conditions }\end{array}$ & $\mathrm{R}_{1}$ & $\mathrm{~V}_{1}$ & $\mathrm{R}_{2}$ & $\mathrm{~V}_{2}$ \\
\hline 1 & $(3.0,0)$ & $(0,0.40)$ & $(1,0)$ & $(0,-1)$ \\
2 & $(3.0,0)$ & $(0,0.40)$ & $(1,0)$ & $(0,1)$ \\
3 & $(2.0,0)$ & $(0,0.95)$ & $(-1,0)$ & $(0,-1)$ \\
4 & $(1.4,0)$ & $(0,0.86)$ & $(-1,0)$ & $(0,-1)$ \\
\hline
\end{tabular}

"Configuration \& phase space of He atom" http://www.indjst.org

(2)

The above two equations are dimensionless, which can be solved numerically for different initial configurations.

Numerical technique

The equations (1) and (2) are solved using RungeKutta IV order coupled with adaptive step size algorithm. When an electron moves very close to the nucleus, its acceleration becomes very large and a very small time step $h$ is required to solve the equations of motion. Hence, it is not efficient to use the same small time step throughout the simulation and instead a variable time step or an adaptive step size algorithm is suggested (Gould \& Tobochnik, 1996). An adaptive step-size algorithm can be implemented as follows.

1. Given a set $f(t)$ of positions and velocities at time $t$, use the current value of $h$ to compute the set $f_{1}(t+h)$ of positions and velocities at time $t+h$.

2. Given the same set $f(t)$ of positions and velocities at time $t$ as in step (1), use half the current value of $h$ to compute the set $\mathrm{f}(\mathrm{t}+\mathrm{h} / 2)$ of positions and velocities at time $t+h / 2$. Then use the set $f(t+h / 2)$ and time step $h / 2$ to obtain a second set $f_{2}(t+h)$ of positions and velocities.

3. Use $f_{1}(t+h)$ and $f_{2}(t+h)$ to compute the difference $\Delta E$ in the total energy.

If $\Delta \mathrm{E}$ is too large i.e., greater than $10^{-5}$, then reduce the time step by a factor of two and repeat steps 1 to 3 . If $\Delta \mathrm{E}$ is too small, i.e., less than $10^{-7}$, then increase the time step by a factor of two. If $\Delta \mathrm{E}$ is acceptable i.e., $10^{-7} \leq$ $\Delta \mathrm{E} \leq 10^{-5}$, then retain the same time step. In the last two

Table 1 Initial conditions for simulation of configuration space and phase space of the electrons . 
Fig. 1. Configuration space (torus) of electrons in helium atom for various initial conditions
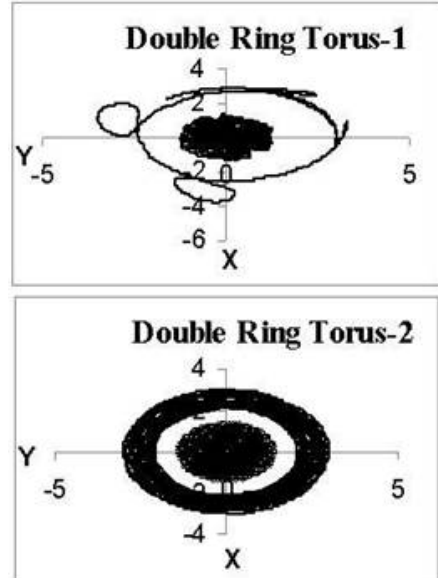
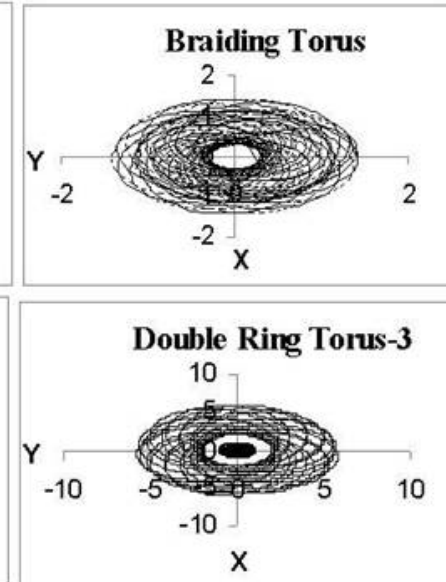

is listed in Table 1. Of these, the simulation of initial conditions-1, 2 and 3 result in double ring torus (Type A) and initial condition-4 results in braiding torus (Type B) as shown in Fig.1. For all the initial conditions the velocity vs position graphs (phase space) are drawn and shown in Fig. 2. The configuration space curves are drawn for various perturbation parameters $(0.3,0.5$, and 0.7$)$ for the initial condition 3 and shown in Fig.3. And the time of escape of electron from helium atom is determined for various perturbation parameters. The auto ionization curve is drawn between the time of escape and perturbation parameter and shown in Fig.3.

\section{Type A: Double ring torus}

If two electrons are distant, with the orbits close to two ellipses, the dynamics is approximated by two Kepler motions. Tori of this type exist even when the angular momenta of the two electrons are opposite.

\section{Type B: Braiding torus}

When the electrons are very close to the nucleus, a novel type of stable orbit is found. This type of torus exists, when $R_{1}=1.40$ and $V_{1}=0.86$. Here the closure of orbits is identical for each electron. Only the phases of two orbits are different. This type of torus has a finite measure in the phase space, and can be semi-classically quantized by suitably choosing their positions. Here two electrons must satisfy a delicate phase relationship; otherwise one of the electrons is kicked away to infinity. In each orbit an electron revolves twice in the inner side, while the other revolves once at the outer circle, before they exchange their position.

\section{The phase space}

The Phase space provides us the additional information on the dynamics of the system under consideration. It is found that, for all the cases $X$ component and the $\mathrm{Y}$-component of phase space showed
Fig.2. Phase space of electrons in helium atom for various initial conditions

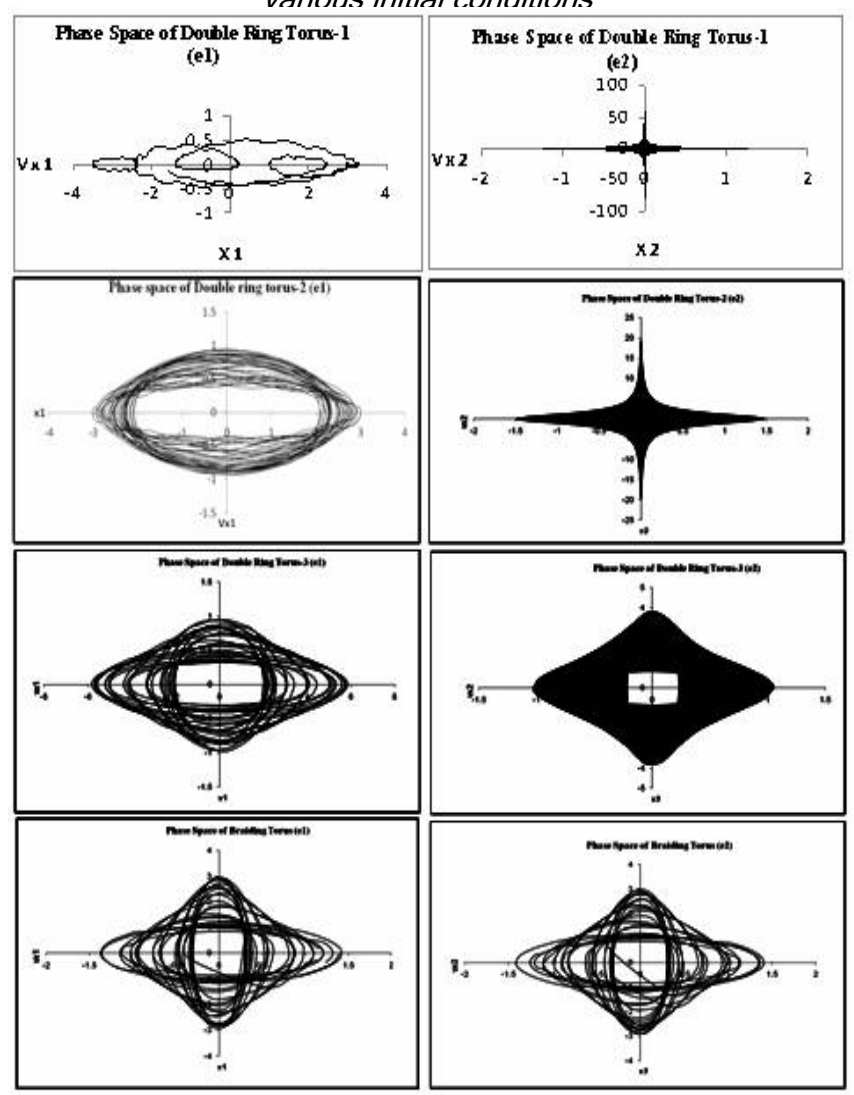

the same behavior and therefore only the X-component of phase space are considered.

In all the cases, at $x=0, v_{x}$ is maximum and vice versa. For the initial conditions $1-3$, the phase of the electrons $\mathrm{e} 1$ and e2 are different while for the initial conditions 4, the phase space appears same for both the electrons. The condition 4 corresponds to the braiding of two electrons path in real space. Since they are in the same torus with a phase difference, the phase space for the two electrons is same.

The phase space for double ring torus-3 (e2) is very interesting. In this case, the electron behaves in a peculiar way. There are two distinct regions; the central square piece, which is completely forbidden and the outer portion, which is completely filled. This leads to semiclassical quantization of torus in phase space.

\section{Perturbation parameter}

The ratio between the two radii (small to large one) plays the major role of perturbation parameter in the Kolmogorov-Arnold-Moser (KAM) theory (Poschel, 2001). When the ratio between the two radii is far from unity, the orbit is again approximated by two ellipses modulated quasiperiodically.

When the perturbation grows, deformation of electron orbits would take place. As the perturbation is large (two electrons lie very close to each other), the torus orbit is
Research article

CIndian Society for Education and Environment (iSee)
"Configuration \& phase space of He atom" http://www.indjst.org
Santhanam et al. Indian J.Sci.Technol. 
deformed more strongly. Simulation had been carried out for condition 3 by varying the perturbation parameter.

(i) When perturbation parameter is 0.3 , the electrons do not interact strongly; each electron revolves in its torus and forms a double ring torus.

(ii) When perturbation parameter is 0.5 , the first electron escaped to infinity, while the second electron moves around the nucleus in a chaotic manner. This results in

Fig.3. Configuration space for different perturbation parameter \& resulting auto-ionization of electrons in helium atom

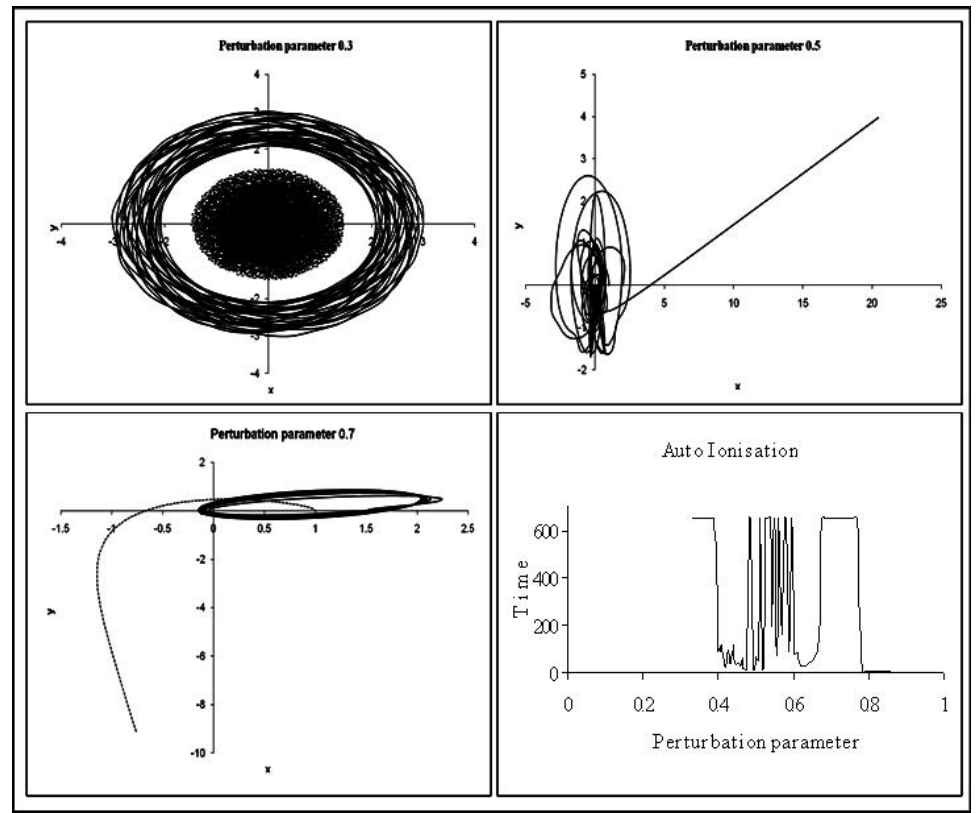

auto-ionization of helium atom.

(iii) When perturbation parameter is 0.7 , the second electron escaped to infinity, whereas the first electron wanders around the nucleus in ellipsoidal torus. This also results in auto-ionization of helium atom.

\section{Auto ionization}

The periodic orbits show a chaotic behavior by any slight change of initial conditions and lead to an escape of one electron implying "auto ionization". All chaotic orbits lead to auto ionization. After some chaotic transients, one of the electrons goes away to infinity. Here, ionization time has been computed as a function of radius for the type-A configuration (condition-3) by fixing the ratio of initial velocity $V_{1} / V_{2}$, and varying the ratio of distance $R_{1} / R_{2}$. If the total energy of the electron is negative, it is bounded with the nucleus and when it becomes positive it escapes out of the nucleus. This is called auto ionization. If the energy of the system is positive over a given duration of time, the atom is regarded to be ionized. The auto ionization time of the system is calculated by varying the perturbation parameter from 0.3 to 0.9 in steps of 0.02. Strong auto ionization takes place when the perturbation parameter lies between 0.4 and 0.7 .

\section{Conclusion}

In the present paper, novel types of torus motions in the classical helium atom with configuration and phase space were discussed. The phase space for double ring torus-3 (e2) shows two distinct regions; the central square piece, which is completely forbidden and the outer portion, which is completely filled. This leads to semiclassical quantization of torus in phase space. For the perturbation parameter values $(0.4$ to 0.7$)$, the ionization is predominant. Even though the system is classical, the phase space shows allowed and forbidden bands which is unique for quantum mechanics. This result is fascinating and needs for further investigation.

\section{References}

1. Bohr N (1913) On the Constitution of Atoms and Molecules. Phil. Magz. 6(26), 1-25.

2. Gould H and Tobochnik J (1996) An introduction to computer simulation methods: Application to physical systems. Addision Wesley, $2^{\text {nd }}$ Ed.

3. Langumuir I (1921) The structure of the helium atom. Phys. Rev. 17, 339-353.

4. Poschel J (2001) A lecture on the classical kamtheorem. Proc. Symp. Pure Maths (AMS). 69, 707732.

5. Van Vleck JH (1922) The dilemma of the helium atom. Phil. Magz. 44, 842-869.

6. Yamamota T and kaneko K (1993) Helium atom as a classical three-body problem. Phys. Rev. Lett. 70, 1928-1931.
"Configuration \& phase space of He atom" http://www.indjst.org
Santhanam et al. Indian J.Sci.Technol. 